Research Article

\title{
New Method for OC Relay Coordination
}

\author{
Ameer Aqeel Kamoona $\mathbb{D D}^{1}{ }^{1}$ Ahmed Najm alfadli, ${ }^{2}$ and Israa Ali Alshabeeb ${ }^{1}$ \\ ${ }^{1}$ Computer System Department, Babylon Technical Institute, Al-Furat Al-Awsat Technical University, 51015 Babylon, Iraq \\ ${ }^{2}$ Ministry of Education, 66001 Al Muthanna, Iraq \\ Correspondence should be addressed to Ameer Aqeel Kamoona; amer@atu.edu.iq
}

Received 26 January 2020; Revised 24 February 2020; Accepted 16 March 2020; Published 13 May 2020

Academic Editor: Herve Morel

Copyright $\odot 2020$ Ameer Aqeel Kamoona et al. This is an open access article distributed under the Creative Commons Attribution License, which permits unrestricted use, distribution, and reproduction in any medium, provided the original work is properly cited.

\begin{abstract}
Protection relays design and manufacture have arrived to a new level of advancement; hence, numerical relays have appeared recently. They combine all the functions of monitoring, control, and protection in one device. Also these relays have the ability to communicate with each other to share statuses and orders using GOOSE (generic object-oriented substation events) messages based on communication protocols such as IEC61850. In this paper, a proposed method for overcurrent (OC) relay coordination has been introduced based on numerical relay features of several setting groups and communication capability. This work supposes that the coordination between feeder, bus section, and incomer protection relays, in a substation of single busbar configuration with bus sectionalizer (i.e., bus section), depends on the status of the bus section circuit breaker (CB) as a criterion for determining active relay setting group, to achieve a faster tripping action. ETAP has been used as a simulation software environment. And the simulation results show the advantages of the proposed method in reducing the time needed by the relay to clear the electrical fault. Siemens SIPROTEC series have been used as an example of numerical relays, specifically 7SJ64 as OC relay, which exists in the ETAP library.
\end{abstract}

\section{Introduction}

After the ingress of microprocessors and operating software in the manufacturing of devices that monitor, control, and protect power system networks, new so-called smart grids have emerged. Numerical relays are the most important part of the smart girds, where they perform a superior function that was achieved by several apparatuses previously, such as their ability to communicate with each other to provide a fully controlled substation. Numerical overcurrent (OC) relay has the traditional function of the $\mathrm{OC}$ relay in detecting the fault condition but it is faster, more selective, and able to perform several functions like fault recording [1].

OC protection is considered as a main protection relay for feeder protection in high voltage substations of different types. The electrical substations can be classified based on many considerations, one of which is the busbar configuration; the one that has been deemed in this work is a single busbar with bus sectionalizer (i.e., bus section) substation which is shown in Figure 1. This configuration is generally used in $132 / 33 / 11 \mathrm{kV}$ substation for 33 and $11 \mathrm{kV}$ side. It has the advantage of increasing system reliability through maintaining the power flow during any defect, by closing the bus section to provide load continuity [2]. Smart grids with the integration of numerical protection lead to new methods for protection setting and coordination; in [3] a distribution network with central controller was considered to communicate with IEDs based on IEC 61850, providing an adaptive setting to relays to mitigate the changes in system configuration. Paper [4] proposed to change the predetermined relays setting groups in order to adapt the relays coordination with the distribution generators (DGs) connection state. In [5], the researchers present a method that finds the optimal settings for relays to obtain adaptive relays and thus face the system significant changes with the help of IEC 61850 based communications. In [6] the authors aimed to propose a hardware-in-the-loop online adaptive method to make the relay setting interchangeable with power system change due to DG connection. The work presented in [7] aims to increase the relay adaptive to more system states by 


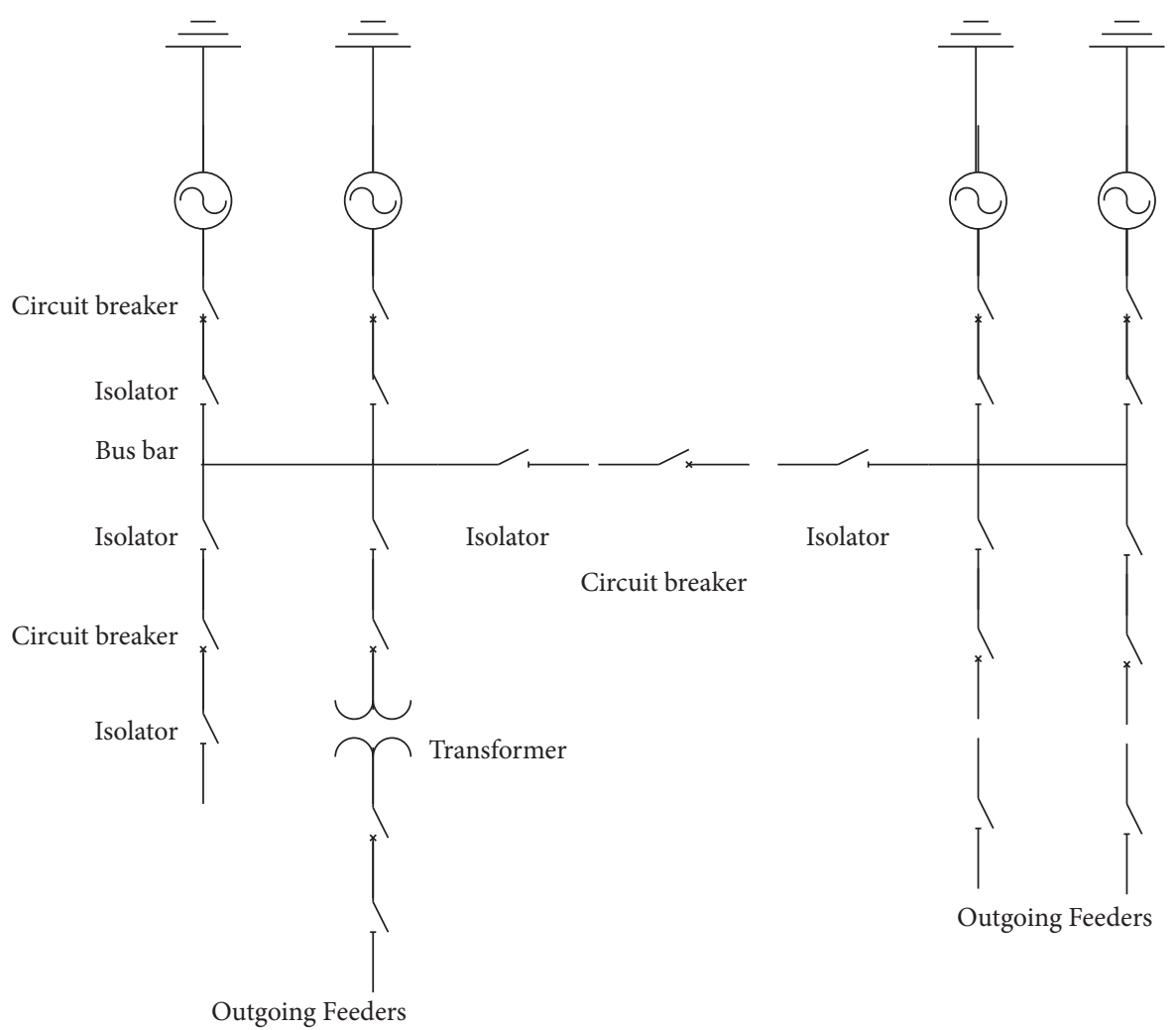

FIgURE 1: Single busbar arrangement with bus sectionalized.

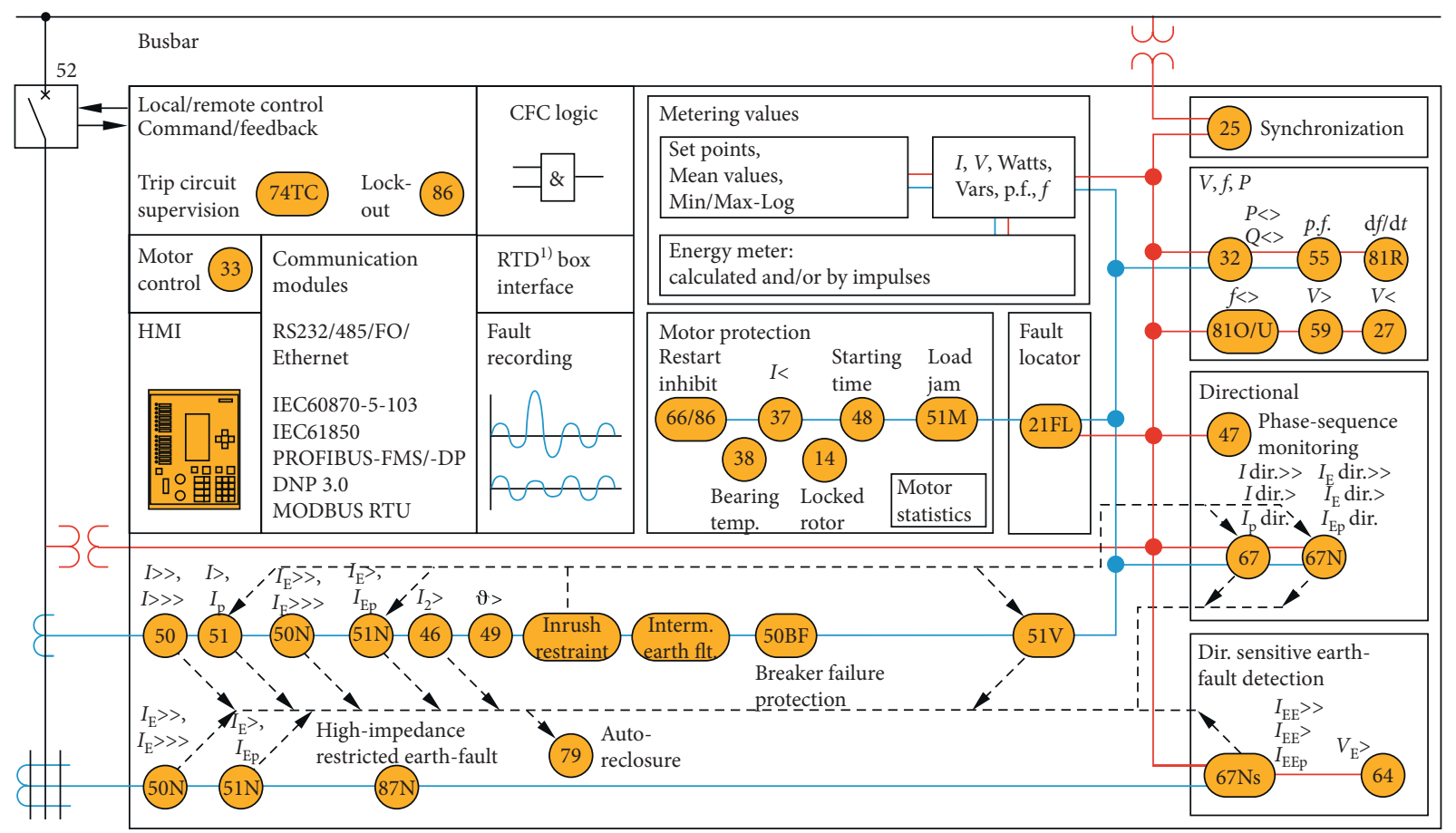

${ }^{1)} \mathrm{RTD}=$ resistance temperature detector

FIGURE 2: 7SJ64 relay function diagram.

change the parameters of the active relay setting with the aid of available communication systems rather than using limited number of setting groups. Reference [8] used the advanced features of the smart grid to mitigate the impact of DG on the distribution network and the advantage of the fuzzy rule based on the calculation of a predetermined relays 


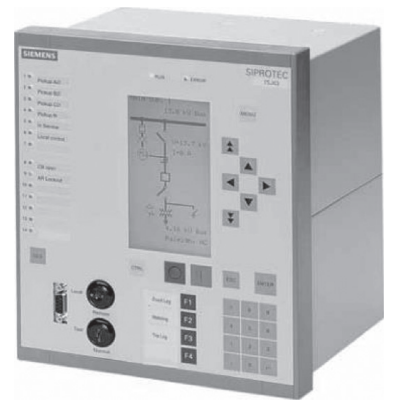

FIGURE 3: Siemens 7SJ64 numerical relay view.

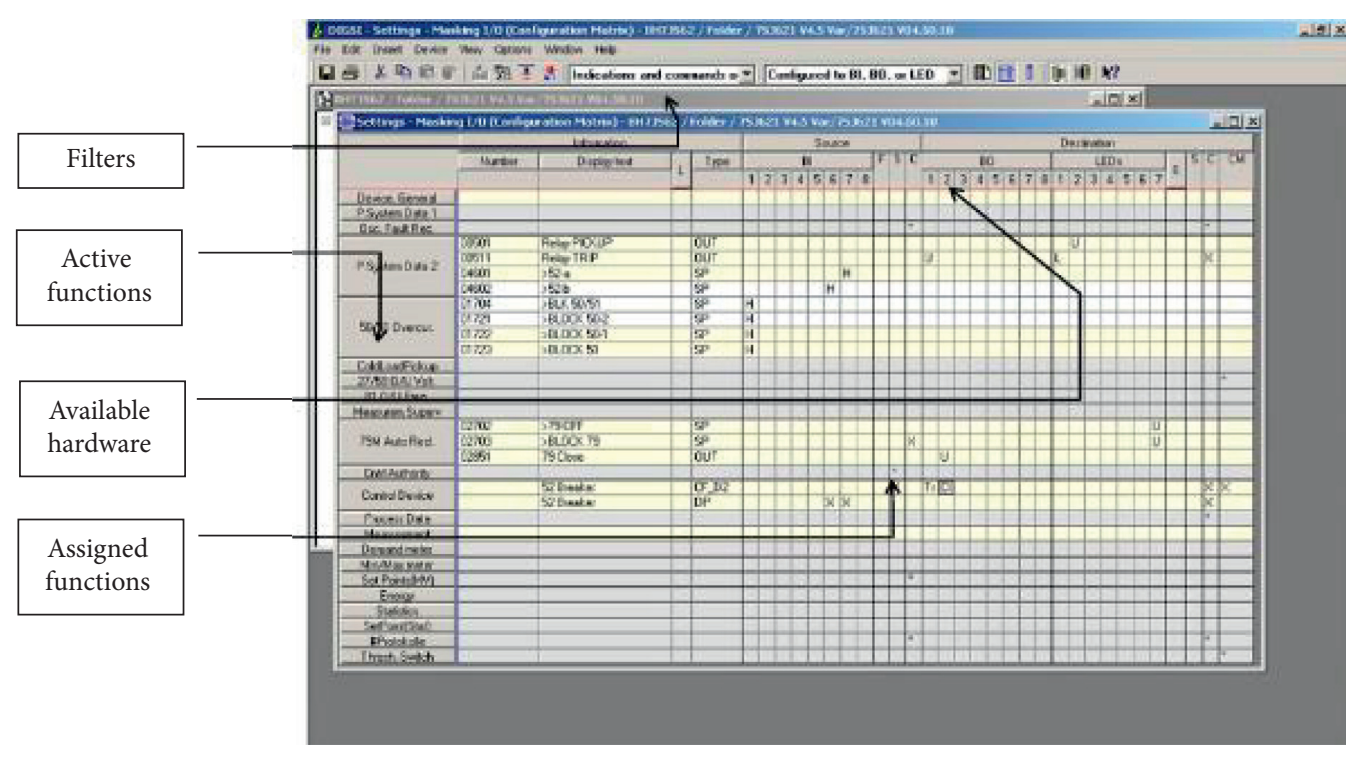

FIGURE 4: DIGSI software.

setting groups, where the genetic algorithm was used to solve the relay coordination problems due to the presence of DGs. In another study [9], the authors consider changing in relays setting groups in order to adapt relays coordination to different system configurations resulting from DG connection; a hybrid genetic algorithm and linear programming method have been used to classify each system configuration into the few number of available relay setting groups. Papers [10-12] present a new method for protection coordination based on different optimal algorithms. The first one depends on the Lightning Flash Algorithm in order to provide OC relay coordination in distribution system, find the best TMS and PS for the relay, and compare the obtained results with other algorithms (PSO and GA).

The second one follows the Nature-Inspired Root Tree Algorithm in order to reduce the time needed by the relay to clear the fault. The last one considers a redial network to coordinate the OC relays based on the Genetic and Particle Swarm Optimization. In [13], the researchers propose a method for the protection of the microgrid rely in the IEC 61850 GOOSE messages to overcome the coordination difficulties due to multiple power sources, and the proposed method has been verified by a testing system. Another study [14] suggested an adaptive method by changing the setting groups of the relays via binary input depending on DGs connection state, for only DGs close relays based on the proposed sensitivity matrix. In [15], the authors investigate the effect of DG on the coordination of the directional overcurrent relay, so they proposed a method for adaptive relay setting based on a microgenetic algorithm and advanced numerical relay monitoring feature to change relay settings.

In this article a new method for numerical OC relay coordination in a single busbar system with bus section has been presented; since the proposed method depends on the IEC 61850 communication protocol between IEDs (Intelligent Electronic Devices) which represent the protection relays, the new coordination method basically depends on the numerical relays feature of the ability to deal with multiple setting groups and assign the active group according to specific input signals.

\section{Numerical Relays SIPROTEC Series}

Siemens Company has a new generation of protection devices "SIPROTEC series", which include many types of protection relays: distance, differential, breaker failure, OC, etc. There are different versions of relays in the series of SIPROTEC 4; the one that is considered here, 7SJ64 relay, is 


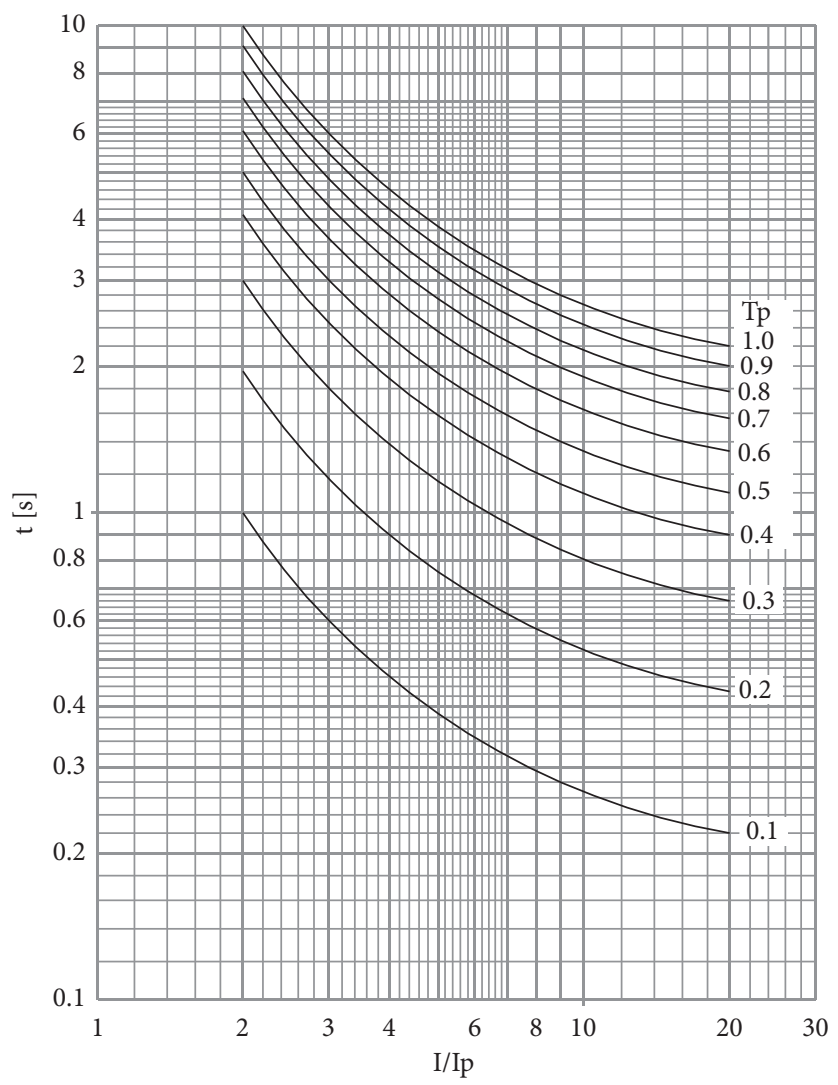

Figure 5: Time and PSM curves, normal inverse acc. to IEC [17].

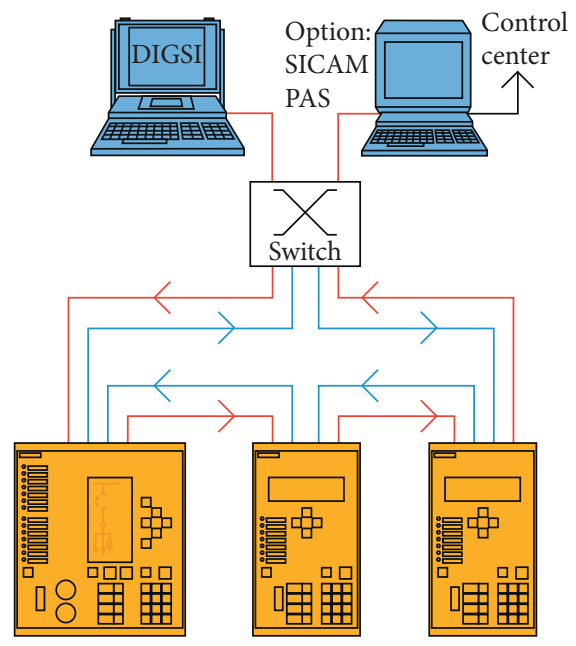

Figure 6: Bus structure for station bus with Ethernet and IEC 61850.

designated for OC protection in distribution and transmission network; moreover, it has the function of monitoring and controlling nearly up to 20 functions, Figure 2 shows the relay function diagram and relay input from the power system measuring and switching equipment. The relay has many input-output binary elements, through which relay can receive and send orders from and to other devices, and the binary element numbers differ from model to the other [16]. Also this relay has the ability to adapt with the system condition by changing its active setting group during relay operation. Switching between setting groups may happen due to internal relay decision or external system device via the binary input or serial communication protocol.

Figure 3 shows the front view of the 7SJ64 relay, as it is clear that the relay has a front display for visual interaction. 


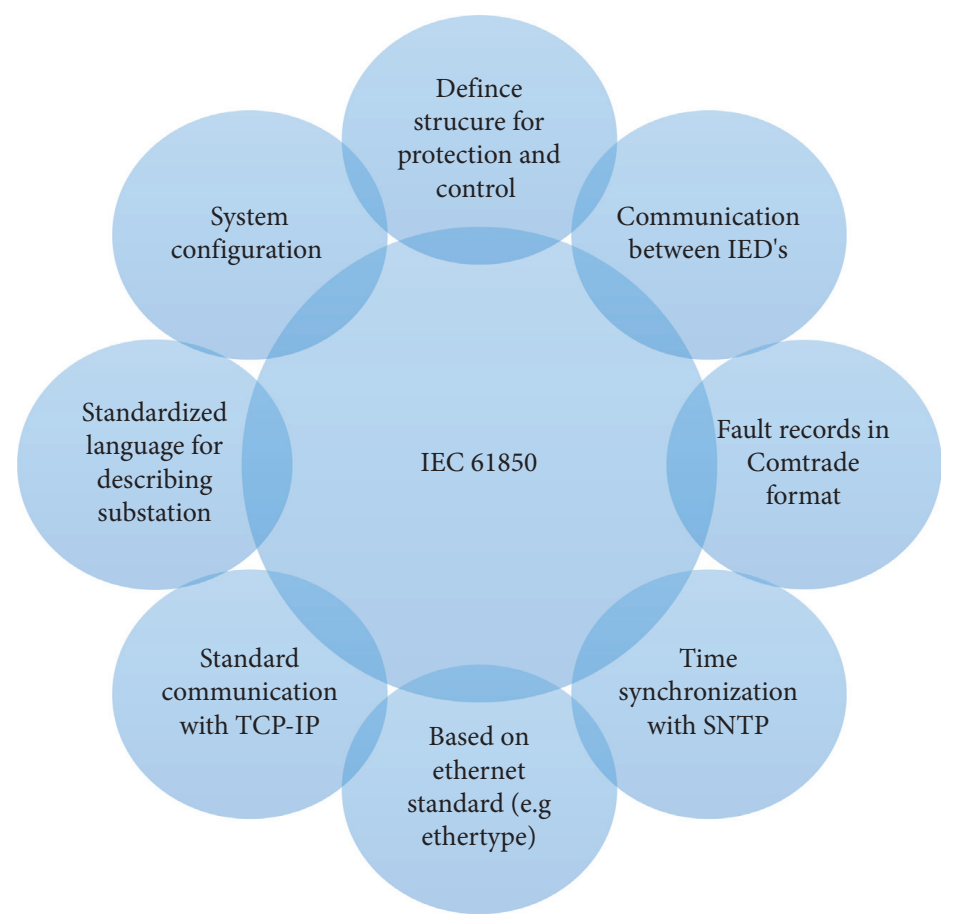

FiguRE 7: IEC61850 application.

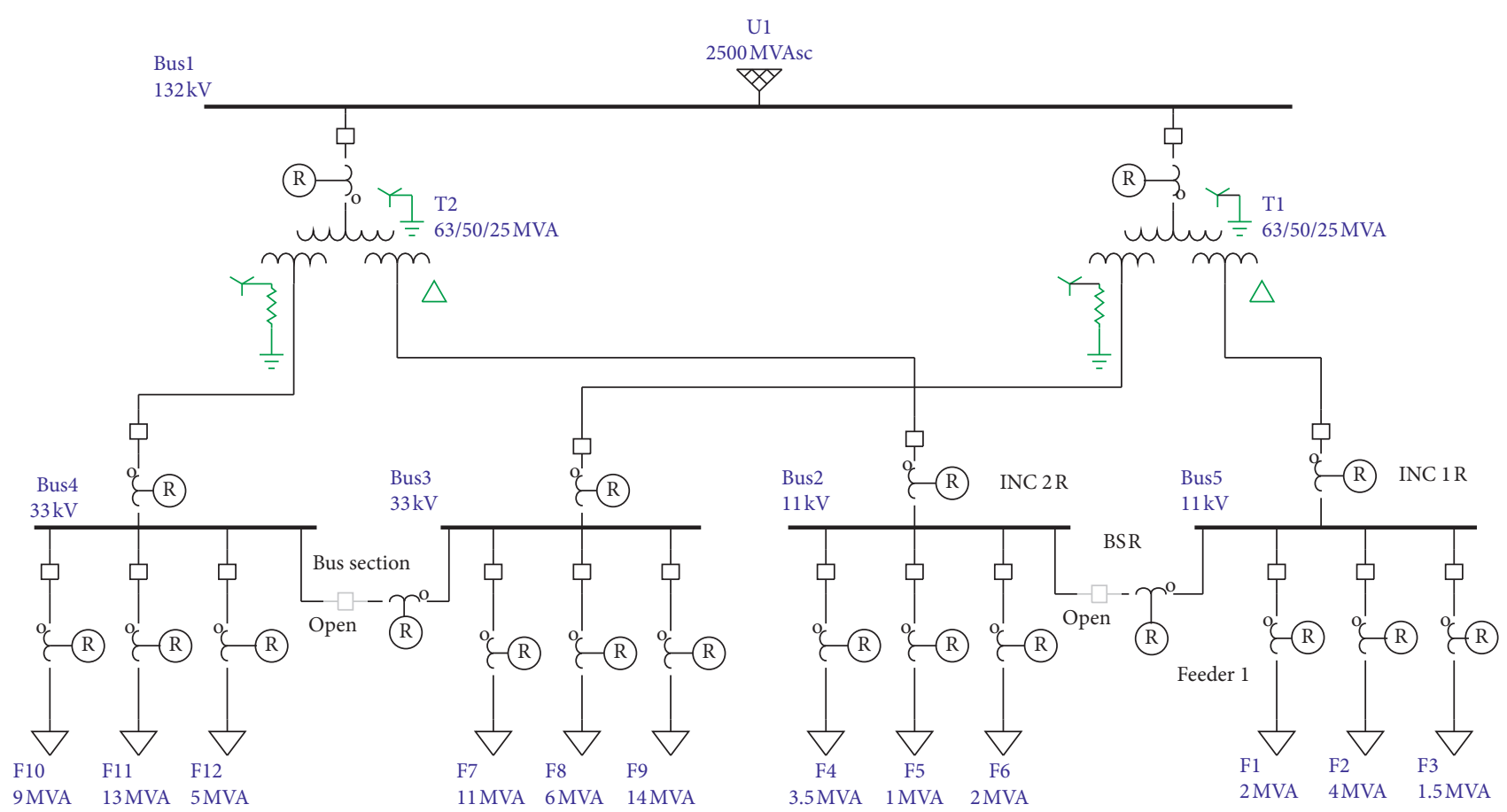

FIGURE 8: The study system, two transformers of $132 / 33 / 11 \mathrm{kV}$ substation.

The relay rear side contains communication ports to contact the other system devices via Ethernet-based IEC61850 protocol which will be explained in detail in the next section.
The configuration of the numerical SIPROTEC relays may be done through the computer with the aid of the socalled DIGSI software, which permits the user to set, 


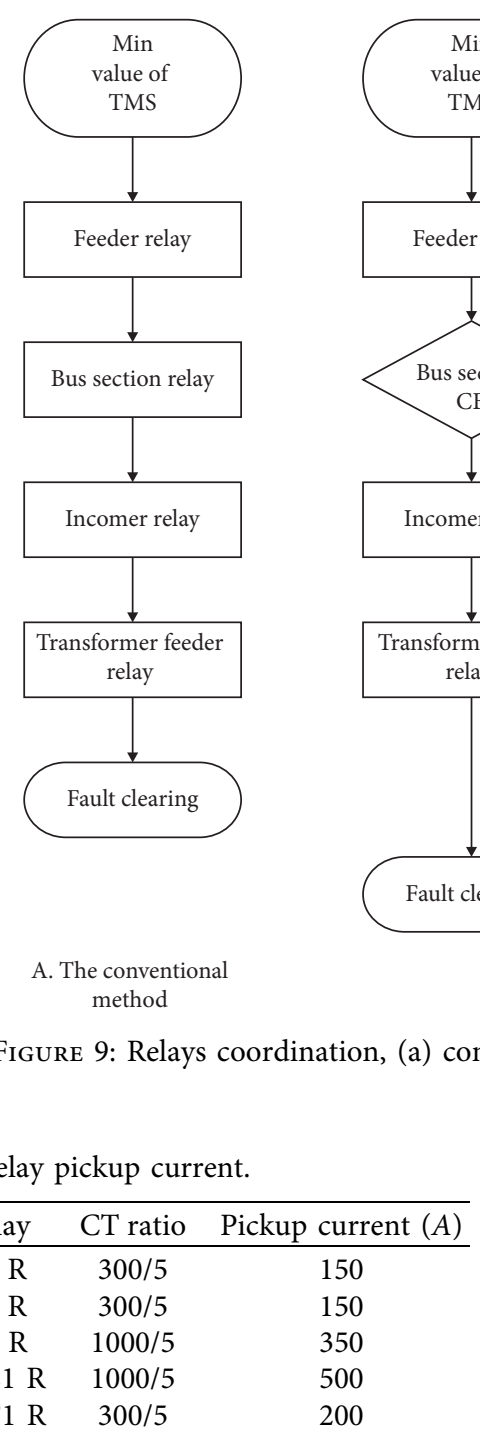

commission, control, and route the numerical relays by very easy human-machine interface software as shown in
Figure 4. For the setting and coordination of the OC relay, plug setting (PS) and time multiplier setting (TMS) are needed for each relay in the system. PS depends on the max load and can be determined by a load flow study, while TMS is computed based on the discrimination time margin needed between the relays.

The following equation is used for setting calculation of the OC relay of the normal inverse characteristics, and Figure 5 shows the relay time-current curve.

$$
\begin{aligned}
& \operatorname{PSM}(\text { plug setting multiplier })=\frac{\text { fault current }}{\text { plug setting } \times \text { rated current of CT }} \\
& \text { Actual operation time }(t)=\frac{0.14}{(\mathrm{PSM})^{0.02}-1} \times \text { TMS. }
\end{aligned}
$$

Or time-PSM curve can be used for the relay to find the time and then find the actual relay time as follows:

$$
t=\text { time from curve } \times \text { TMS. }
$$

Time margin is $0.2-0.4 \mathrm{sec}$ which is used to provide the selectivity between relays. 


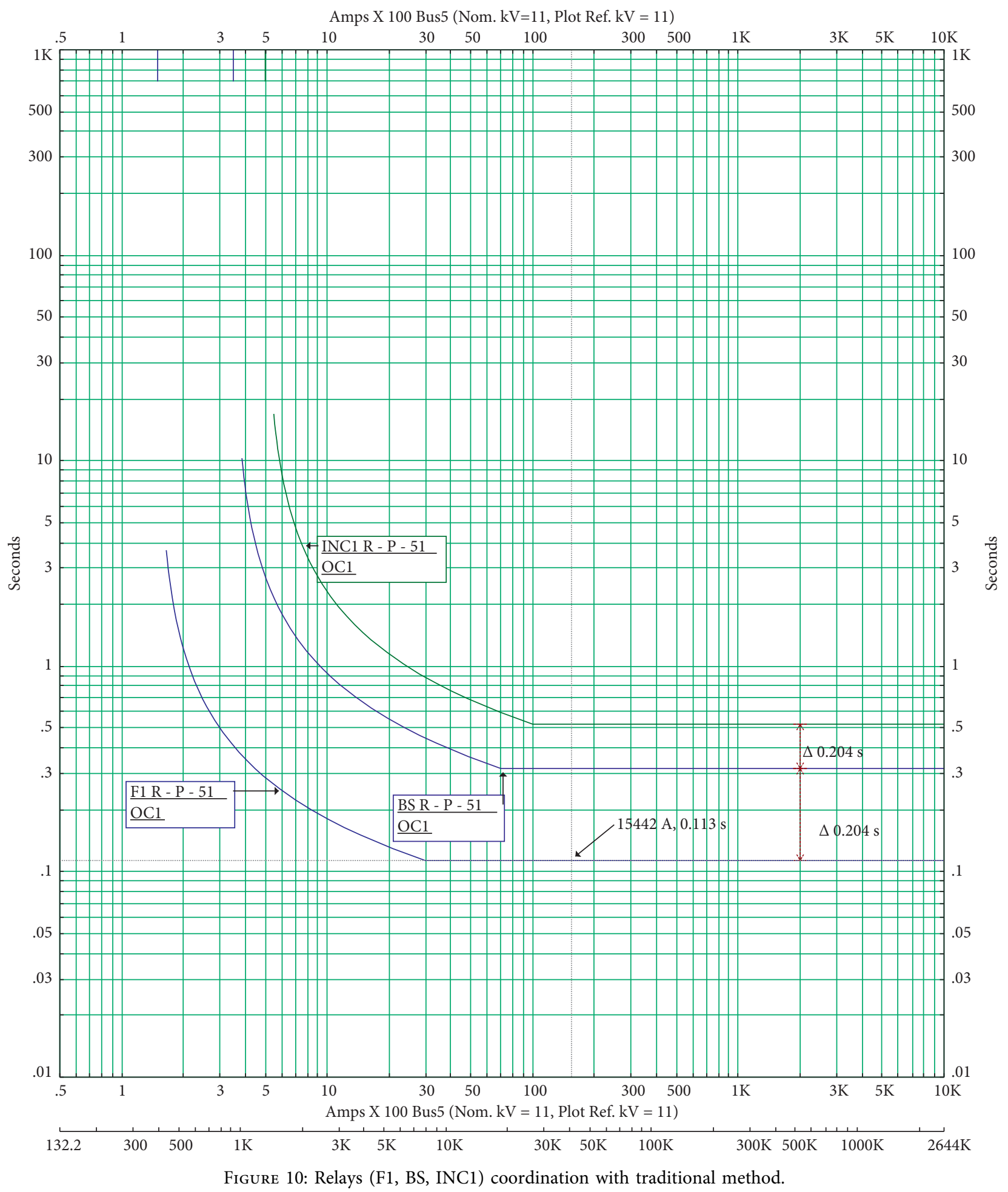

TABLE 2: Relays operation time for feeders 1 and 6 fault, with traditional method.

\begin{tabular}{lccc}
\hline Relay & TMS & $\begin{array}{c}\text { Bus section CB OFF } \\
\text { Operation time } t(\mathrm{sec}) \text { for F1 fault }\end{array}$ & $\begin{array}{c}\text { Bus section CB ON } \\
\text { Operation time } t \text { (sec) for F6 fault }\end{array}$ \\
\hline F1 R & 0.05 & 0.113 & $\infty$ \\
F6 R & 0.05 & $\infty$ & 0.113 \\
BS R & 0.14 & $\infty$ & 0.317 \\
INC1 R & $\mathbf{0 . 2 3}$ & $\mathbf{0 . 5 2 1}$ & $\mathbf{0 . 5 2 1}$ \\
\hline
\end{tabular}




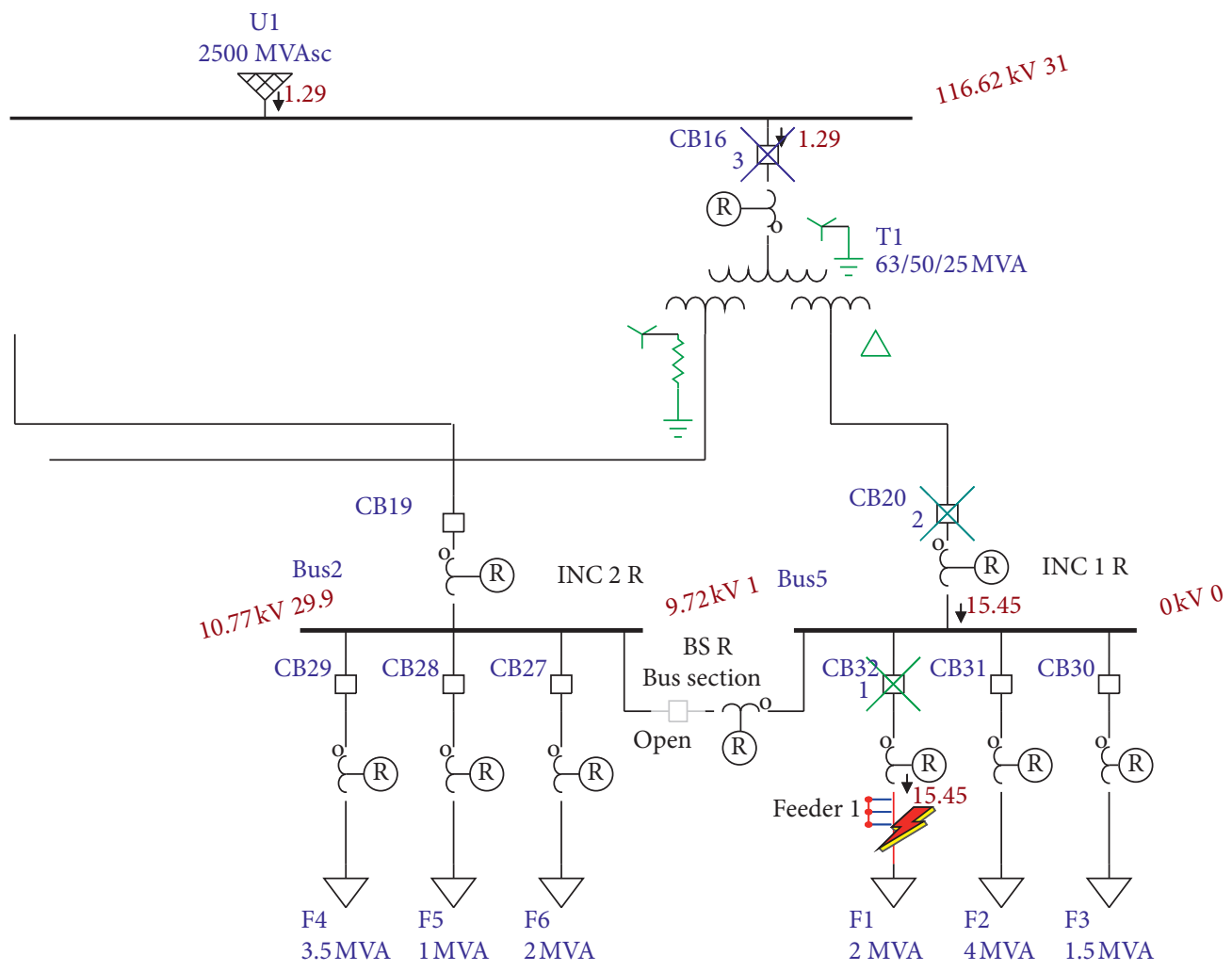

FIgURE 11: Relays operation sequence for feeder 1 fault with bus section CB, OFF.

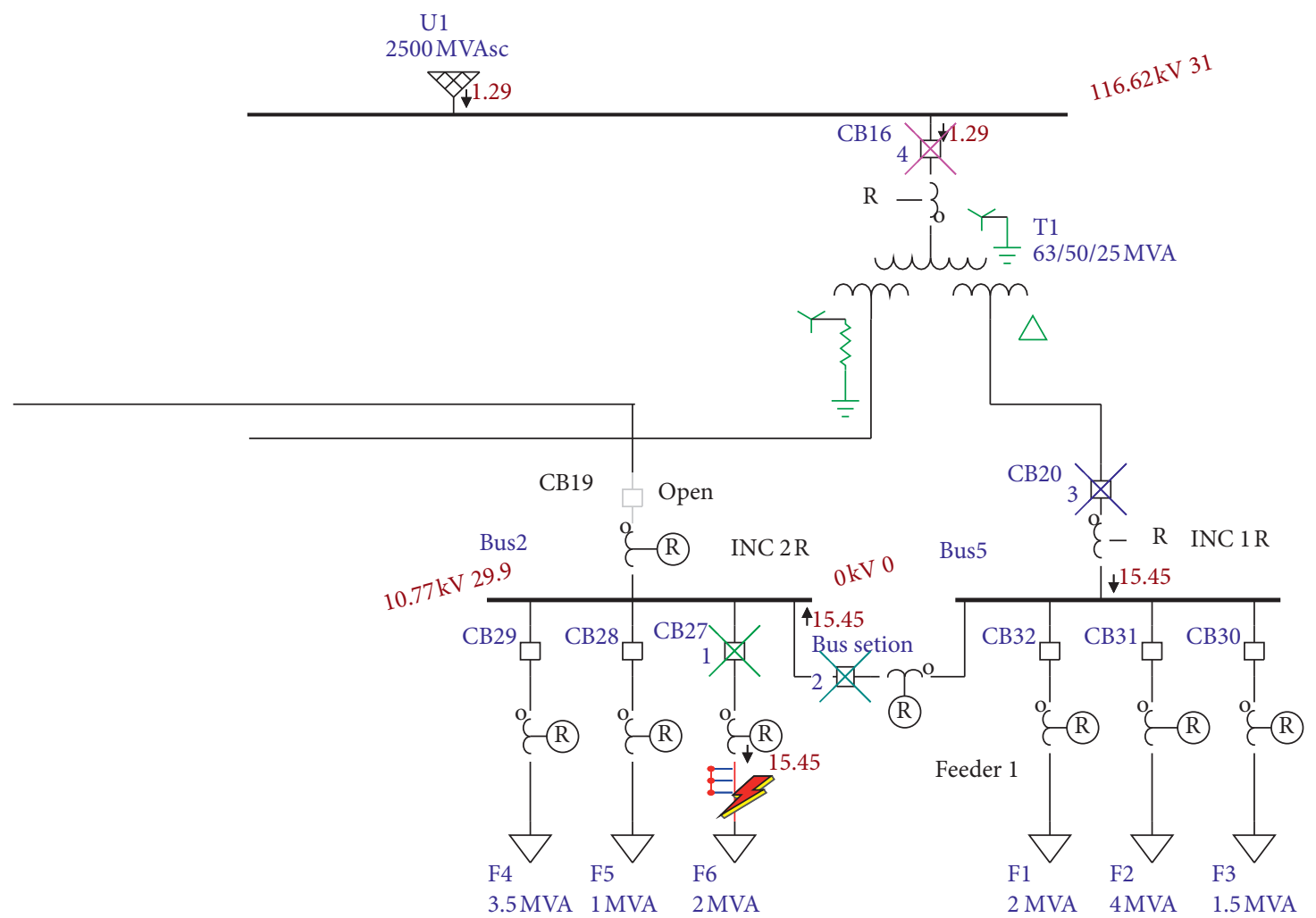

Figure 12: Relays operation sequence for feeder 6 fault with bus section CB, ON. 
TABLE 3: Relays operation time for feeders 1 and 6 fault, for the proposed method.

\begin{tabular}{|c|c|c|c|c|c|}
\hline \multirow[t]{2}{*}{ Relay } & \multicolumn{2}{|r|}{ Bus section $\mathrm{CB}$ OFF } & \multirow[t]{2}{*}{ Relay } & \multicolumn{2}{|r|}{ Bus section $\mathrm{CB}$ ON } \\
\hline & TMS & Operation time $t(\mathrm{sec})$ for $\mathrm{F} 1$ fault & & TMS & Operation time $t$ (sec) for F6 fault \\
\hline F1 R & 0.05 & 0.113 & F1 R & 0.05 & $\infty$ \\
\hline F6 R & 0.05 & $\infty$ & F6 R & 0.05 & 0.113 \\
\hline BS R & 0.14 & $\infty$ & BS R & 0.14 & 0.317 \\
\hline INC1 R “group B” & 0.14 & 0.317 & INC1 R "group $A$ ” & 0.23 & 0.521 \\
\hline
\end{tabular}

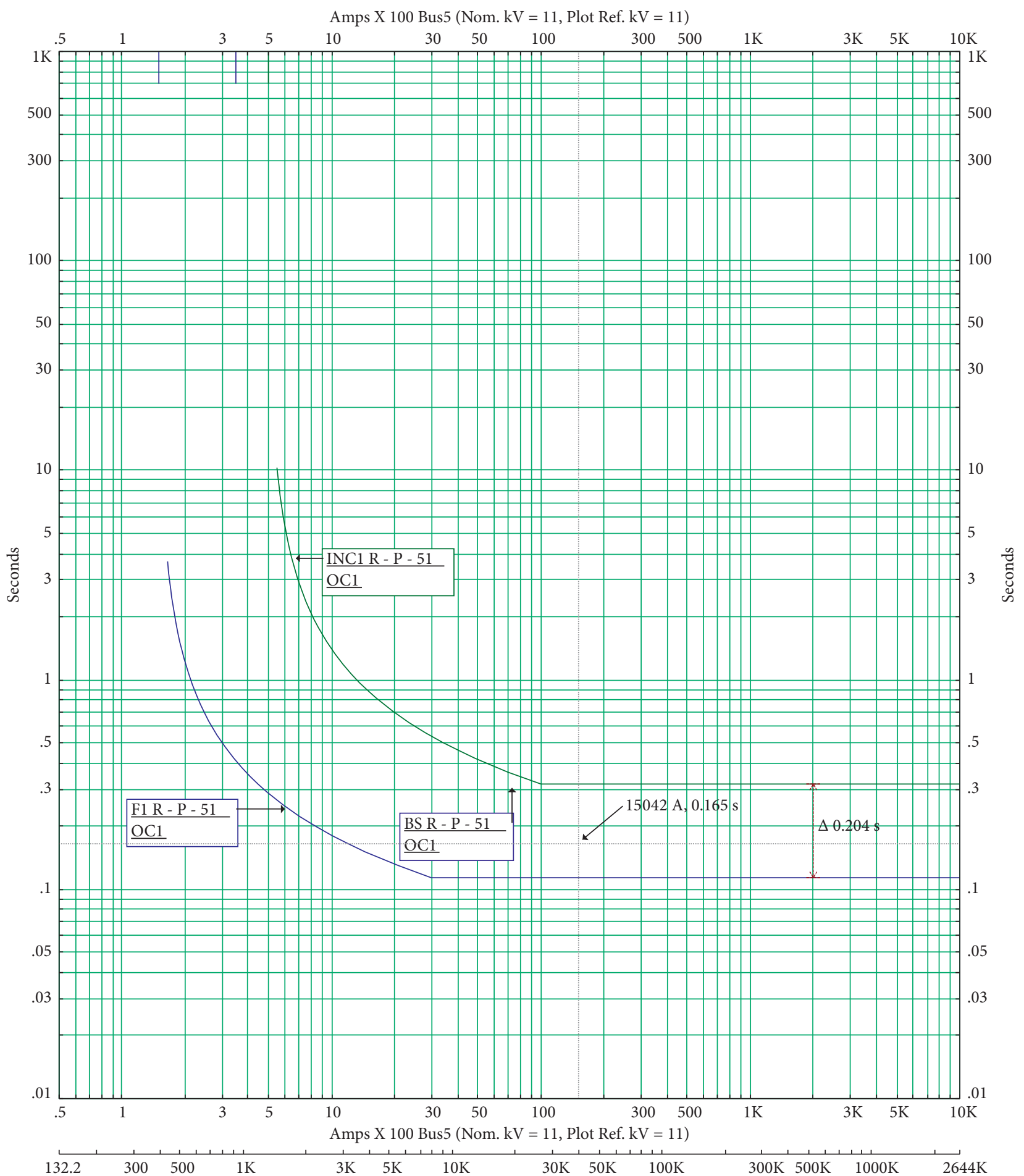

Figure 13: Relays (F1, BS, INC1) coordination with the proposed method, INC1 R group " $B$ ". 


\section{Relay Communication Protocol IEC 61850}

The advancement in the technology led to more reliable and easier communication between protection relays themselves and with the central substation controller. One of the most famous and usable worldwide communication protocols is the IEC 61850 standard. With this protocol, data can be transferred directly among bays to provide system interlocks. Figure 6 shows the connection between relays and system controller through Ethernet-IEC61850 protocol, since the relays are capable of sending "GOOSE" (generic object-oriented substation events) messages for peer-to-peer relay communications $[18,19]$.

The use of the IEC61850 protocol brings several advantages, especially in reducing the cost, since the applications that were dependent on wires connection and auxiliary contactors are now performed though the LAN connection resulting in high speed achievement. Figure 7 shows an example of the IEC61850 applications [20].

\section{Conventional versus Proposed Methods for OC Relay Coordination}

As mentioned before the new "proposed" method of OC coordination basically depends on two advanced features of the numerical relays; they have the ability to communicate with each other via the communication protocol IEC 61850 and multiple setting groups, and the active setting group can be decided by an order through the communication protocol control. Let us consider a system of single busbar with bus section configuration as in Figure 8 in order to explain the proposed method; this figure shows a single line diagram of a $132 / 33 / 11 \mathrm{kV}$ substation with the protection relays for each part (i.e., feeder (F), incomer (INC), bus section (BS), and transformer feeder (TRF)). With this type of busbar configuration, when one transformer gets out of service for any reason (like internal fault or maintenance), the bus section circuit breaker (CB) can be set to ON state to maintain the power continuity to the feeders from the other in-service transformer.

First let us start to show the conventional method of relays coordination; feeder relay (F R) TMS must be set to the minimum applicable value, and then $\mathrm{BS}$ relay will get the next value of TMS depending on the safety margin; the grading in TMS continues through the INC relay till TRF relay which is considered as the last backup protection. As it is clear that the old method of coordination did not take into account the bus section CB status, whether it is in service or not, this will lead to delay in the operation of INC relay as backup protection.

The proposed method of coordination considers the bus section $\mathrm{CB}$ status, since when it is out of service, which is the most operable situation, there is no need to consider the BS relay in the coordination to speed up the operation of the INC relay. This can be achieved by making the INC relay of an adapted setting (i.e., has two setting groups) for the incomers of both transformers at either side of BS; the active setting group depends on the status of the bus section $\mathrm{CB}$, which is sent to the INC relays by a GOOES message via the IEC 61850 communication link that connects all substation relays together. If the bus section $\mathrm{CB}$ gets $\mathrm{ON}$, then INC relay active setting group can be " $A$ " which makes the relays coordination same as in the traditional method, or else the active setting group is " $B$ " which speeds up the operation of the INC relay by neglecting the BS relay in coordination, since the TMS grading will start form F relay toward INC relay and does not take into account the BS relay. Figure 9 shows a flowchart of the TMS grading for the proposed relay coordination method versus the conventional one.

\section{Simulation Results}

In order to verify the advantage of the proposed coordination method, ETAP software has been used to simulate the study system of Figure 8. All system relays have been coordinated using ETAP with the aid of the relays pickup current in Table 1; the data in this table is only for the verification related process relays at $11 \mathrm{kV}$ side. 7SJ64 Siemens numerical protection relay has been used in the simulation, which already exists in the ETAP library. There are no stated details about the relay setting calculation, because this paper contains a relay coordination study and does not consider relay setting procedure.

The simulation fault analysis considers a 3-phase fault at $11 \mathrm{kV}$ feeders 1 and 6 (i.e., transformers 1 and 2), with two cases of BS, OFF and ON, respectively. For the conventional method, there is only one coordination procedure with the two BS cases as shown in Figure 10. Table 2 shows relays operation time and Figures 11 and 12 indicate the relay operation sequence for the two above-mentioned cases at fault condition.

Now, regarding the proposed method of coordination, when bus section CB is ON, this makes INC relay be in the group " $A$ " of setting, since in this case the relay coordination and operation time are similar to those in the traditional method of coordination in Figure 10. Table 3 shows relays TMS and operation time for F6 fault. However, in the case when bus section $\mathrm{CB}$ is OFF, the INC relay will activate group " $B$ " of setting which will lead to different relay coordination as in Figure 13 and get the speed up in the INC relay operation time for F1 fault as shown in Table 3.

Its clear form the INC relay operation time results in Tables 2 and 3 that there is an improvement in the relay time and, hence, a reduction in the relay operating time exists in the proposed method of coordination.

\section{Conclusion}

The development in power system generation, transmission, and distribution is much accelerated in the fields of control, monitoring, and protection; on the other hand, our protection strategies must be changed in compliance with this development. Consequently, this study utilizes the new features of numerical protection relays for updating the protection relays coordination procedure. The proposed method has been applied for a substation of $132 / 33 / 11 \mathrm{kV}$ using ETAP simulation software, and the results show a reduction of about $0.2 \mathrm{sec}$ in the operating time of the INC relay due to the usage of the multiple setting groups 
mechanism, in which less time is needed by the relay to clear the fault that in roll, has a direct impact on the high voltage expensive apparatus (i.e., transformers, CBs, etc.) live time. The proposed method can be applied for different substation configurations and must give an improvement in the relays coordination and operation time.

\section{Data Availability}

The ETAP software data used to support the findings of this study are available from the corresponding author upon request.

\section{Conflicts of Interest}

The authors declare that they have no conflicts of interest.

\section{References}

[1] S. Kannuppaiyan and V. Chenniappan, "Numerical inverse definite minimum time overcurrent relay for microgrid power system protection," IEEE Journal Transactions on Electrical and Electronic Engineering, vol. 10, no. 1, pp. 50-54, 2015.

[2] S. Kamel, S. Ahmed, K. Ahmed, L. Nasrat, J. Yu, and S. Wang, "Short circuit analysis and coordination of overcurrent relays for a realistic substation located in upper Egypt," in Proccedings of the 2019 IEEE Innovative Smart Grid TechnologiesAsia (ISGT Asia), pp. 2150-2155, IEEE, Perth, Australia, November 2019.

[3] D. Ishchenko, A. Oudalov, and S. James, "Protection coordination in active distribution grids with IEC 61850," in Proccedings of the PES T\&D, pp. 1-6, IEEE, Montevideo, Uruguay, September 2012.

[4] M. Khederzadeh, "Adaptive setting of protective relays in microgrids in grid-connected and autonomous operation," in Proccedings of the 11th IET International Conference on Developments in Power Systems Protection (DPSP 2012), p. 14, Birmingham, UK, April 2012.

[5] F. Coffele, C. Booth, and A. Dyśko, "An adaptive overcurrent protection scheme for distribution networks," IEEE Transactions on Power Delivery, vol. 30, no. 2, pp. 561-568, 2014.

[6] V. A. Papaspiliotopoulos, G. N. Korres, V. A. Kleftakis, and N. D. Hatziargyriou, "Hardware-in-the-loop design and optimal setting of adaptive protection schemes for distribution systems with distributed generation," IEEE Transactions on Power Delivery, vol. 32, no. 1, pp. 393-400, 2015.

[7] M. Ojaghi and V. Mohammadi, "Use of clustering to reduce the number of different setting groups for adaptive coordination of overcurrent relays," IEEE Transactions on Power Delivery, vol. 33, no. 3, pp. 1204-1212, 2017.

[8] El Naily, S. M. S. Naser, T. Hussein, K. El-Arroudi, and F. A. Mohamed, "On-line adaptive protection scheme to overcome operational variability of DG in smart grid via fuzzy logic and genetic algorithm," in Proceedings of the 2018 9th International Renewable Energy Congress (IREC), pp. 1-6, IEEE, Hammamet, Tunisia, March 2018.

[9] R. M. Chabanloo, M. Safari, and R. G. Roshanagh, "Reducing the scenarios of network topology changes for adaptive coordination of overcurrent relays using hybrid GA-LP," IET Generation, Transmission \& Distribution, vol. 12, no. 21, pp. 5879-5890, 2018.

[10] M. Kheshti and X. Kang, "Optimal overcurrent relay coordination in distribution network based on Lightning Flash
Algorithm," Engineering Computations, vol. 35, no. 3, pp. 1140-1160, 2018.

[11] A. Wadood, S. Gholami Farkoush, T. Khurshaid et al., "An optimized protection coordination scheme for the optimal coordination of overcurrent relays using a nature-inspired root tree algorithm," Applied Sciences, vol. 8, no. 9, p. 1664, 2018.

[12] M. Lakshmi, R. D. Narayanasamy, N. Banerjee, and O. V. Gnana Swathika, "Optimum coordination of overcurrent relays using genetic algorithm and Particle Swarm optimization," SSRN Electronic Journal, https://papers.ssrn.com/sol3/JELJOUR_ Results.cfm?form_name=journalbrowse\&journal_id=3219840\& Network $=$ no\&lim $=$ false, 2019.

[13] J.-C. Gu, C.-H. Liu, J.-M. Wang, and M.-Ta Yang, "Using IEC 61850 GOOSE messages in microgrid protection," International Transactions on Electrical Energy Systems, vol. 29, 2019.

[14] A. Abbasi, H. K. Karegar, and T. S. Aghdam, "Adaptive protection coordination with setting groups allocation," International Journal of Renewable Energy Research (IJRER), vol. 9, no. 2, pp. 795-803, 2019.

[15] J. P. Nascimento, N. S. D. Brito, and B. A. Souza, "An adaptive overcurrent protection system applied to distribution systems," Computers \& Electrical Engineering, vol. 81, p. 106545, 2020.

[16] A. Marian, M. Adam, M. Andrusca, A. Dragomir, and C. N. Deac, "Aspects regarding the protection relays from power substations," in Proceedings of the 2019 International Conference on Electromechanical and Energy Systems (SIEL$M E N)$, pp. 1-6, IEEE, Craiova, Romania.

[17] Manual-SIPROTEC Multi-Functional Protective Relay with Local Control 7SJ62/63/64 V4.6, 2005.

[18] B. Duncan and S. Howard, "Applications and advantages for protection schemes using IEC 61850 standard," in Proceedings of the 2006 Power Systems Conference: Advanced Metering, Protection, Control, Communication, and Distributed Resources, pp. 63-72, IEEE, Clemson, South Carolina, March 2006.

[19] S. Kimura, A. Rotta, R. Abboud, R. Moraes, E. Zanirato, and J. Bahia, "Applying IEC 61850 to real life: modernization project for 30 electrical substations," in Proceedings of the 10th Annual Western Power Delivery Automation Conference, Spokane, WA, USA, April 2008.

[20] I. Ali and M. S. Thomas, "GOOSE based protection scheme implementation \& testing in laboratory," in Proceedings of the ISGT, pp. 1-7, IEEE, Sarajevo, Bosnia-erzegovina, October 2011. 